\title{
Urban green and grey space in relation to respiratory health in children
}

\author{
Christina Tischer ${ }^{1,2,3}$, Mireia Gascon ${ }^{1,2,3}$, Ana Fernández-Somoano 3,4 , \\ Adonina Tardón ${ }^{3,4}$, Aitana Lertxundi Materola ${ }^{5,6}$, Jesus Ibarluzea $3,6,7$, \\ Amparo Ferrero 3,8 , Marisa Estarlich ${ }^{3,8}$, Marta Cirach ${ }^{1,2,3}$, Martine Vrijheid ${ }^{1,2,3}$, \\ Elaine Fuertes ${ }^{1,2,3}$, Albert Dalmau-Bueno ${ }^{1,2,3}$, Mark J. Nieuwenhuijsen ${ }^{1,2,3}$, \\ Josep M. Antó $1,9,2,3$, Jordi Sunyer ${ }^{1,9,2,3}$ and Payam Dadvand ${ }^{1,2,3}$
}

Affiliations: ${ }^{1}$ Campus MAR, Barcelona Institute for Global Health (ISGlobal), Barcelona, Spain. ${ }^{2}$ Universitat Pompeu Fabra (UPF), Barcelona, Spain. ${ }^{3}$ CIBER Epidemiology and Public Health (CIBERESP), Madrid, Spain. ${ }^{4}$ Instituto Universitario de Oncología del Principado de Asturias (IUOPA), Dept of Medicine, University of Oviedo, Asturias, Spain. ${ }^{5}$ Universidad del Pais Vasco (UPV)/Euskal Herriko Unibertsitatea (EHU), Leioa, Spain.

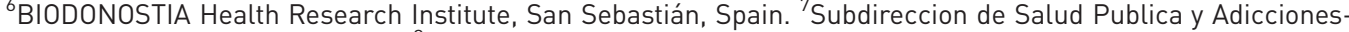
Gipuzkoa, San Sebastián, Spain. ${ }^{8}$ Joint Research Unit for Epidemiology and Environmental Health, FISABIOUniversitat de València-Universitat Jaume I, Valencia, Spain. ${ }^{9}$ Institut Hospital del Mar d'Investigacions Mèdiques (IMIM), Barcelona, Spain.

Correspondence: C. Tischer, Campus MAR, Barcelona Institute for Global Health (ISGlobal), Dr Aiguader 88, 08003 Barcelona, Catalonia, Spain. E-mail: christina.tischerdisglobal.org

@ERSpublications

Associations between respiratory health and urban residential greenness and greyness differ by geographic region http://ow.ly/pnGJ30bqBn1

Cite this article as: Tischer C, Gascon M, Fernández-Somoano A, et al. Urban green and grey space in relation to respiratory health in children. Eur Respir J 2017; 49: 1502112 [https://doi.org/10.1183/ 13993003.02112-2015].

ABSTRACT We assessed the effect of three different indices of urban built environment on allergic and respiratory conditions.

This study involved 2472 children participating in the ongoing INMA birth cohort located in two biogeographic regions (Euro-Siberian and Mediterranean) in Spain. Residential surrounding built environment was characterised as 1) residential surrounding greenness based on satellite-derived normalised difference vegetation index (NDVI), 2) residential proximity to green spaces and 3) residential surrounding greyness based on urban land use patterns. Information on wheezing, bronchitis, asthma and allergic rhinitis up to age 4 years was obtained from parent-completed questionnaires. Logistic regression and generalised estimating equation modelling were performed.

Among children from the Euro-Siberian region, higher residential surrounding greenness and higher proximity to green spaces were negatively associated with wheezing. In the Mediterranean region, higher residential proximity to green spaces was associated with a reduced risk for bronchitis. A higher amount of residential surrounding greyness was found to increase the risk for bronchitis in this region.

Associations between indices of urban residential greenness and greyness with respiratory diseases differ by region. The pathways underlying these associations require further exploration.

This article has supplementary material available from erj.ersjournals.com

Received: Dec 142015 | Accepted after revision: Feb 252017

Support statement: A full list of the funding bodies and awards that supported the work featured in this article can be found in the Acknowledgements section.

Conflict of interest: None declared.

Copyright OERS 2017 


\section{Introduction}

Paediatric asthma and allergy are prevalent conditions worldwide and environmental factors are thought to play a key role in their development $[1,2]$. How exposure to green areas in residential urban environments may influence the development of these conditions has recently received increased attention. It has been speculated that greenness may help to reduce the prevalence of allergic conditions by enhancing the biodiversity of the living environment, which, in turn, may be inversely associated with immune system dysfunctions, such as atopy [3-5]. In fact, an inverse association between higher greenness around the residential address and a lower risk of atopic sensitisation has been observed especially in rural areas characterised by less densely built-up area and more native vegetation [6]. Such an association is less clear in populations from (sub-)urban environments. Recent studies in paediatric populations reported inconsistencies between exposures to urban green spaces in relation to allergic outcomes [7-12]. These inconsistencies might reflect differences in the study setting, such as climate and geographic characteristics [13]. Further, urban environments are known to vary in their "grey" surfaces, which comprise industrial, transport and urban-fabric characteristics. These factors are thought to potentially influence the environmental profile towards a "dysbiotic drift" [14]. In this context, a limited body of evidence has suggested that "urbanicity" is associated with severity of wheeze symptoms in infants [15].

The aim of the present study was to prospectively evaluate the association between indicators of urban green and grey built environments and respiratory and allergic health outcomes in young children. Towards this aim we also evaluated whether such associations would vary across different bio-geographic regions [13]. Accordingly, we chose study regions in Spain with distinct climates and vegetation patterns: two study centres in the Euro-Siberian study region (Asturias and Gipuzkoa), characterised by an Atlantic climate with high water availability, and two study centres (Sabadell and Valencia) in the Mediterranean area, which has a predominantly dry climate. To our knowledge, no study has evaluated the impacts of green and grey spaces on asthma and allergic conditions in order to provide a comprehensive perspective on their interrelated health impacts.

\section{Materials and methods}

\section{Study population and study area}

The INMA (INfancia y Medio Ambiente; Environment and Childhood) birth cohort is a network of population-based birth cohorts across Spain, which was set up for the purpose of studying the impact of environmental factors on pregnancy outcomes, child growth and development. Detailed information on the cohorts and data collection processes have been published elsewhere [16]. Our study used data from four INMA study centres: Asturias, Gipuzkoa, Sabadell and Valencia. These four centres are located on the Iberian Peninsula, which encompasses two distinct bio-geographic regions in Spain: the Euro-Siberian region (which contains the Asturias and Gipuzkoa study centres) and the Mediterranean region (which contains the Sabadell and Valencia study centres). The Euro-Siberian region covers a narrow ridge across the northern part of the peninsula and is characterised by an Atlantic climate with year-round high water availability, relatively cold winters, and maximum vegetation during the summer months. The rest of the peninsula is considered Mediterranean, and is characterised by a dry climate with hot and dry summers, mild and rainy winters, and maximum vegetation during autumn and spring. These two regions have distinct climates and vegetation patterns (figure 1) [18, 19].

FIGURE 1 Infancia y medio ambiente (INMA) birth cohorts and biogeographic regions across the Iberian Peninsula (based on data from [17]).

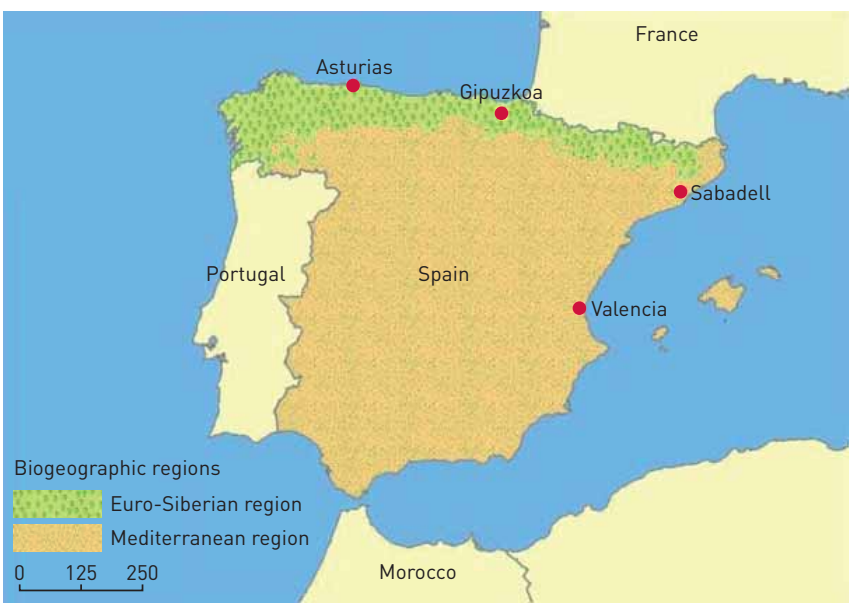


Exposure assessment

We used three different indices of built environment: two different measures of urban green space exposure, namely residential surrounding greenness and residential proximity to green spaces, and a measure of non-natural built-up infrastructure, namely residential surrounding greyness. Residential surrounding greenness is a surrogate for general outdoor greenness of the living environment, while residential proximity to green spaces is a surrogate for access to green spaces. These two measures can be considered to be "complementary" as surrounding greenness does not necessarily imply access to green space $[7,20]$. Further, in order to cover urban land use characteristics, we included residential surrounding greyness as a surrogate for the non-natural built-up infrastructures.

\section{Residential surrounding greenness}

The assessment of residential surrounding greenness was based on the satellite-derived Normalised Difference Vegetation Index (NDVI). The NDVI is an indicator of greenness and is based on land surface reflectance of visible (red) and near-infrared parts of the spectrum [21]. Its values range from -1 to 1 , with higher positive numbers indicating more greenness (i.e. photosynthetically active vegetation). To develop NDVI maps for our study regions, we used Landsat 4-5 Thematic Mapper (TM) data at $30 \mathrm{~m} \times 30 \mathrm{~m}$ resolution [8, 18], which was obtained via the Global Visualization Viewer of the U.S. Geological Survey (2011). The Landsat TM data, describing residential greenness around birth, were acquired for 2007 [18] (2004-2008). For each participant, mean NDVI values in 300-m buffers around the place of residence at the time of birth and at age 4 years were calculated $[8,22]$.

\section{Residential proximity to green spaces}

To determine residential proximity to green spaces, we used the Urban Atlas map developed by the European Environment Agency (2007), which details land use at a scale of 1:10000 for large urban areas (>100000 inhabitants) across Europe [23]. As the Urban Atlas provides data separately on urban green areas, we were able to construct two binary variables (yes/no) that indicated whether each child's residential address at birth and at age 4 years was located within $300 \mathrm{~m}$ of an urban green space. The 300-m distance was selected to accord with the European Commission's general recommendations for access to green space [24].

\section{Residential surrounding greyness}

The CORINE (Coordination of Information on the Environment) programme, developed by the European Union, is a Europe-wide satellite-based inventory of land-cover categorised into 44 classes at a scale of 1:100000 [25], updated in 2006. CORINE land-cover classes are organised into three hierarchical levels (Level 1: five categories; Level 2: 15 categories; Level 3: 44 categories). To determine the residential surrounding greyness, we used the land-cover nomenclature based on Level 2 by summing up associated land use types (expressed in $\mathrm{m}^{2}$ ) within a $300-\mathrm{m}$ buffer around the home address. Residential surrounding greyness was classed as either industrial, commercial and transport units, or mine, dump and construction sites, or urban fabric related features.

\section{Health outcomes at age 4 years}

Wheeze at age 1 year was defined as a positive answer to the following question in the parental questionnaire: "In the last 6 months, has your child ever experienced whistling or wheezing from the chest, but not noisy breathing from the nose?" At age 4 years, wheezing in the past 12 months was assessed as: "Considering the past 12 months, which of the following sentences describes the situation of your child best? Never wheezing (0 episodes), sometimes wheezing (1-2 episodes), often wheezing (3-6 episodes), very often wheezing ( $7-12$ episodes), or wheezing most of the time ( $\geqslant 12$ episodes)?" The variable was recoded into "yes/no wheezing in the past 12 months", with only those reporting never wheezing classified as "no". The occurrence of physician-diagnosed bronchitis was defined as a positive answer to "In the last 6 months (or 12 months if asked at the age of 4 years), has the doctor told you that your child has had bronchitis?" for the study centres in Asturias and Valencia. For Gipuzkoa and Sabadell, bronchitis was assessed through parental reports. In the age-4-year questionnaire, the parents were further asked about the asthma and allergic rhinitis status of their child: "In the past 12 months, has your child ever suffered from asthma (or allergic rhinitis)?" For the INMA Asturias and Valencia study centres, the asthma and allergic rhinitis questions differed slightly: "In the past 12 months, has your child been diagnosed with asthma (or allergic rhinitis)?"

\section{Potential confounders}

Information on each child's sex, maternal education (primary or less, secondary school, and university), maternal allergy, breast feeding, pets at home at birth, maternal smoking during pregnancy and second-hand exposure to tobacco at home at age 4 years was obtained through parent-completed 
questionnaires. To characterise the neighbourhood socioeconomic status (SES) of each study participant, we applied the Urban Vulnerability Index [26] at a census tract level based on 21 indicators of urban vulnerability. For the analysis, we dichotomised the Urban Vulnerability Index using the median as the cut-off: <the median ("less deprived") and $\geqslant$ the median ("more deprived"). We applied area-specific land use regression (LUR) models to estimate exposure to nitrogen dioxide $\left(\mathrm{NO}_{2}\right)$ at the residence during pregnancy and in the first year of life. A detailed description of assessment of air pollution exposure can be found elsewhere [27].

\section{Statistical analysis}

Analyses were performed for the entire study population as well as after stratification by bio-geographic region (Euro-Siberian and Mediterranean). As NDVI measurements around birth and around the age of 4 years were highly correlated (Spearman's correlation coefficient $\geqslant 0.94$ ), they were averaged and labelled as life-course exposure to residential surrounding greenness. As the same was true for proximity to green spaces, we also created a life-course variable for this exposure. Exposure to residential surrounding greenness (NDVI) was categorised into tertiles for the total and stratified analyses because not all functional relationships between NDVI and the health outcomes appeared linear. The same was true for residential surrounding greyness.

Binomial logistic regression models using the glm () function with a logit link [28] were applied to analyse the associations between exposure to urban green and grey space with asthma and allergic rhinitis at age 4 years.

\section{Longitudinal analysis}

As information was gathered at two time points for wheezing and bronchitis ( 1 and 4 years of age), we used generalised estimating equations to model these exposure-health relationships. These models are recommended for longitudinal data with repeated measurements as they take into account the incidence and remission of symptoms over a certain time period $[29,30]$.

All results are presented as adjusted odds ratios (aOR) with corresponding 95\% confidence intervals (95\% CI). All statistical analyses were performed using the R statistical package [31].

For sensitivity analyses, we additionally evaluated the influence of the following information: 1) maternal pre-pregnancy body mass index (BMI), categorised as " $<25$ " and " $\geqslant 25$ "; 2 ) whether the child moved to a new location within the first 4 years of life; 3 ) physical activity outside of school activities at age 4 years (median value used as cut-off: less active $\left(<12 \mathrm{~h} \cdot \mathrm{week}^{-1}\right)$ versus more active $\left(\geqslant 12 \mathrm{~h} \cdot\right.$ week $\left.^{-1}\right)$ ) and 4$) \mathrm{NO}_{2}$ exposure within the first year of life.

\section{Results}

In total, 2472 subjects from two bio-geographic regions in Spain, with complete information on exposure at baseline, were included in this study. Participants from the cohorts in the Euro-Siberian region had higher residential surrounding greenness (Wilcoxon-Mann-Whitney test, $\mathrm{p}<0.001$ ) than those in the Mediterranean region (figure 2 and table 1). Accordingly, within the Euro-Siberian region, the percentage of participants living within $300 \mathrm{~m}$ of a green space was higher than that in the Mediterranean region ( $89 \%$ versus $67 \%$, respectively, Chi-squared test, $\mathrm{p}<0.001$ ). The correlation between the exposure indices can be described as moderate. A correlation table has been provided as supplementary material (see supplementary table S5). These results imply that the three indices can be considered as complementary methods for describing the residential built environment.

The population characteristics of the two distinct bio-geographic areas are outlined in table 2. As loss to follow-up occurred during the study period (up to age 4 years), the actual sample size for each exposureoutcome pair is indicated in tables $3-5$, as well as in the supplementary material.

\section{Associations between green and grey space exposure and respiratory outcomes}

As shown in tables 3-5, no statistically significant associations were found between exposure to residential surrounding greenness, residential proximity to green spaces and residential surrounding greyness in relation to any of the health outcomes tested in the pooled analyses.

\section{Region-specific associations}

Higher residential surrounding greenness (3rd tertile versus 1st tertile) was statistically significantly associated with a lower risk of wheezing up to age 4 years in the Euro-Siberian region (adjusted odds ratio (aOR) $0.61,95 \%$ CI 0.44-0.85), but not in the Mediterranean region (aOR 0.92, 95\% CI 0.70-1.20). In the Euro-Siberian region, higher residential surrounding greenness was associated with increased risk of asthma, but the association was statistically significant only for the second tertile of exposure (2nd tertile 

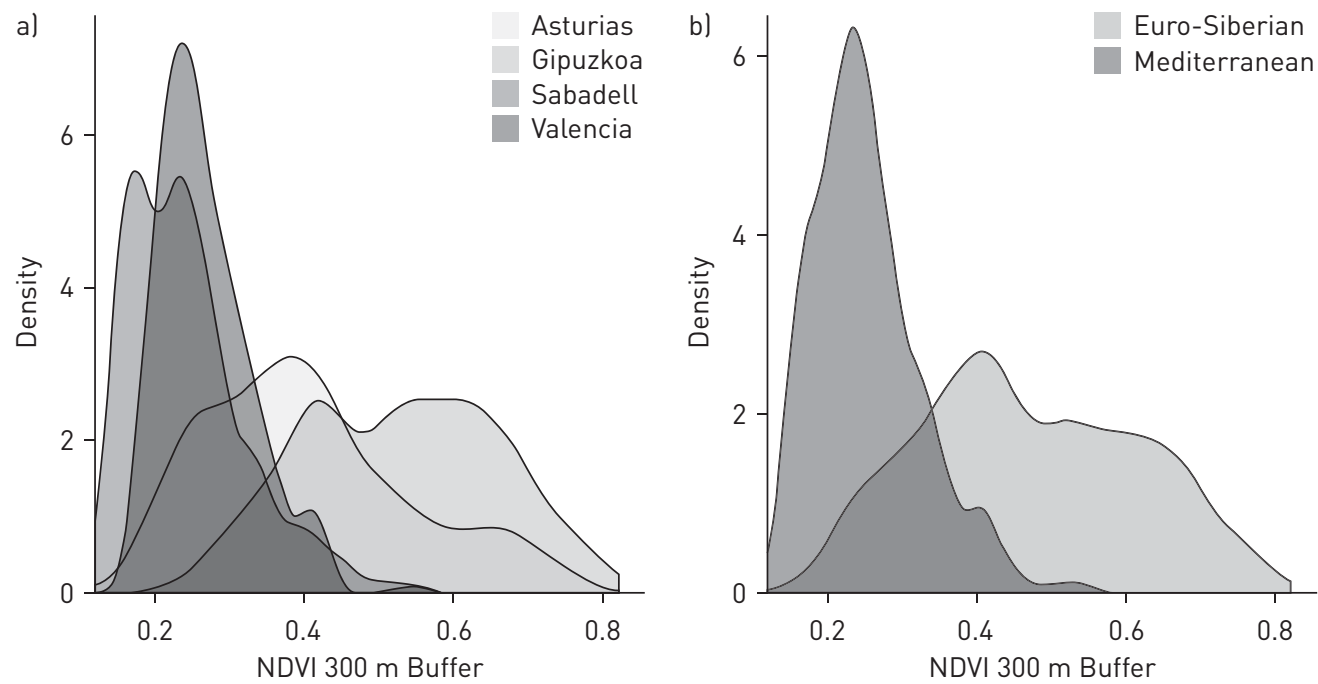

FIGURE 2 Distribution of normalised difference vegetation index (NDVI; 300-m buffer, density plot), stratified by cohort (a) and biogeographic region (b).

versus 1st tertile: aOR 2.46, 95\% CI 1.01-6.00). Residential proximity to green spaces was negatively associated with wheezing in subjects in the Euro-Siberian region (aOR 0.67, 95\% CI 0.45-0.99) and with doctor-diagnosed bronchitis among children in the Mediterranean region (aOR 0.77, 95\% CI 0.61-0.98), as shown in table 4. As presented in table 5, higher exposure to residential surrounding greyness was associated with an increased risk of bronchitis and wheezing for children growing up in the Mediterranean study centres, although the association for wheezing was borderline statistically significant (3rd tertile versus 1st tertile: aOR 1.58, 95\% CI 1.19-2.08 versus aOR 1.29, 95\% CI 0.98-1.70, respectively).

\section{Sensitivity analyses}

After additional adjustment for $\mathrm{NO}_{2}$ exposure at the first year of life, the association between exposure to higher residential surrounding greenness and wheezing was found to be slightly stronger in the Euro-Siberian region (3rd tertile versus 1st tertile: aOR 0.53, 95\% CI 0.37-0.76). For the Mediterranean region, exposure to higher residential surrounding greenness was found to be statistically significantly associated with a reduced risk for bronchitis (3rd tertile versus 1 st tertile: aOR $0.71,95 \%$ CI $0.51-1.00$ ). Additional adjustment for maternal BMI only marginally affected the results for wheezing (3rd tertile versus 1st tertile: aOR $0.62,95 \% \mathrm{CI} 0.45-0.85$ ) and asthma (3rd tertile versus 1 st tertile: aOR $2.45,95 \% \mathrm{CI}$ 1.01-6.00) for subjects growing up in the Euro-Siberian region. Further, for those subjects who had not moved to a new location during the study period, the association between residential surrounding

TABLE 1 Overview and distribution of residential surrounding greenness and greyness exposure measures stratified by biogeographic region.

All cohorts $(n=2472)$

Euro-Siberian ( $n=1108)$

Mediterranean ( $n=1364)$

$\begin{array}{cc}\text { Residential surrounding greenness (ND } \\ \text { Median } & 0.30 \\ \text { 1st tertile } & {[0.120,0.240]} \\ \text { 2nd tertile } & {[0.240,0.389]} \\ \text { 3rd tertile } & {[0.389,0.822]} \\ \text { Residential proximity } & \text { to green spaces m } \\ \text { Median } & 121 \\ \text { Residential surrounding greyness } \mathbf{~ m}^{2} \\ \text { Median } & 193000 \\ \text { 1st tertile } & {[0,134135]} \\ \text { 2nd tertile } & {[134135,250320]} \\ \text { 3rd tertile } & {[250320,282614]}\end{array}$

0.45

$[0.142,0.397]$

$[0.397,0.542]$

$[0.542,0.822]$

68

141300

$[0,103704]$

[103704, 184084]

$[184084,282614]$
0.20

$[0.120,0.204]$

$[0.204,0.265]$

$[0.265,0.570]$

180

241100

$[0,182260]$

[182260, 278422]

[278422, 282614$]$

NDVI: normalised difference vegetation index. 
TABLE 2 Characteristics of the study population stratified by biogeographic region.

\begin{tabular}{|c|c|c|c|c|}
\hline & All cohorts & Euro-Siberian & Mediterranean & p-value (difference) ${ }^{\#}$ \\
\hline Wheezing (1st year) & $833 / 2326(36)$ & 445/1044 (43) & $388 / 1282(30)$ & $<0.001$ \\
\hline Bronchitis (1st year) & $410 / 2320(18)$ & $137 / 1042(13)$ & $273 / 1278(21)$ & $<0.001$ \\
\hline Bronchitis (4 years) & $369 / 1967(19)$ & $122 / 795(15)$ & $247 / 1172(21)$ & $<0.01$ \\
\hline Asthma ( 4 years) & $64 / 1968$ (3) & $42 / 796(5)$ & $22 / 1172(2)$ & $<0.001$ \\
\hline Maternal education & & & & $<0.001$ \\
\hline None/primary & $545 / 2409$ (23) & $171 / 1106(16)$ & $374 / 1303(29)$ & \\
\hline Secondary & $1007 / 2409(42)$ & $442 / 1106(40)$ & $565 / 1303(43)$ & \\
\hline University & $857 / 2409(36)$ & $493 / 1106(45)$ & $364 / 1303(28)$ & \\
\hline Maternal smoking in pregnancy & $414 / 2350(18)$ & $157 / 1040$ (15) & $257 / 1310(20)$ & $<0.01$ \\
\hline Passive smoking (4 years) & $884 / 1978(45)$ & $293 / 808(36)$ & $591 / 1170(51)$ & $<0.001$ \\
\hline Breastfeeding & $1959 / 2299(85)$ & $803 / 990(81)$ & $1156 / 1309(88)$ & $<0.001$ \\
\hline Maternal allergy & $638 / 2466(26)$ & $263 / 1107(24)$ & $375 / 1359$ (28) & 0.03 \\
\hline Maternal BMI & $23(21-25)$ & $22(21-25)$ & $23(21-26)$ & 0.04 \\
\hline Pets at birth & $734 / 2249(33)$ & $245 / 1043(24)$ & $489 / 1206(41)$ & $<0.001$ \\
\hline Physical activity ( 4 years) h-week ${ }^{-1}$ & $12(5-16)$ & $14(10-17)$ & $9(2-15)$ & $<0.001$ \\
\hline Neighbourhood SES ${ }^{+}$(>median) & $1337 / 2299(58)$ & $475 / 1095(43)$ & $862 / 1204(72)$ & $<0.001$ \\
\hline $\mathrm{NO}_{2}$ (1st year) $\mu \mathrm{g} \cdot \mathrm{m}^{-3}$ & $27(20-35)$ & $20(15-25)$ & $34(29-42)$ & $<0.001$ \\
\hline Change of residency (birth to 4 years) & $424 / 2472(17)$ & $138 / 1108(13)$ & $286 / 1365(21)$ & $<0.001$ \\
\hline \multicolumn{5}{|c|}{$\begin{array}{l}\text { Data are presented as } \mathrm{n}, \mathrm{n} / \mathrm{n}(\%) \text { or median (interquartile range) unless otherwise stated. BMI: body mass index. }{ }^{\#} \text { :Chi-squared test and } \\
\text { Wilcoxon-Mann-Whitney test (depending on the variable scale). }{ }^{\Uparrow} \text { :Number based on exposure data. }{ }^{+} \text {:Neighbourhood socioeconomic status } \\
\text { (SES) was determined according to the urban vulnerability index (where, at national level according to } 21 \text { indicators of socioeconomic } \\
\text { vulnerability, } 0=\text { less vulnerable; } 1=\text { vulnerable). }\end{array}$} \\
\hline
\end{tabular}

TABLE 3 Adjusted odds ratios (aORs) ${ }^{\#}$ for the association between life-course residential surrounding greenness (normalised difference vegetation index (NDVI); 300-m buffer) and health outcomes at the age of 4 years, stratified by biogeographic region.

\begin{tabular}{lccc} 
& All cohorts & Euro-Siberian & Mediterranean \\
\hline GEE model & 1777 & & 1048 \\
Wheezing & $1.01(0.82-1.25)$ & $0.88(0.65-1.19)$ & $0.79(0.60-1.03)$ \\
2nd versus 1st tertile & $0.96(0.71-1.30)$ & $0.61(0.44-0.85)$ & $0.92(0.70-1.20)$ \\
3rd versus 1st tertile & 1770 & 723 & 1047 \\
Bronchitis & $1.18(0.95-1.47)$ & $0.98(0.67-1.43)$ & $0.91(0.68-1.20)$ \\
2nd versus 1st tertile & $1.18(0.86-1.62)$ & $0.99(0.67-1.46)$ & $0.95(0.72-1.25)$ \\
3rd versus 1st tertile & 1770 & 724 & 1067 \\
Logistic regression model & $0.82(0.37-1.81)$ & $2.46(1.01-6.00)$ & $1.08(0.32-3.64)$ \\
Asthma & $1.82(0.71-4.67)$ & $2.26(0.91-5.67)$ & $2.05(0.69-6.06)$ \\
2nd versus 1st tertile & 1769 & 722 & 1067 \\
3rd versus 1st tertile & $0.69(0.34-1.43)$ & $0.75(0.28-1.99)$ & $0.84(0.33-2.15)$ \\
Allergic rhinitis & $0.57(0.22-1.50)$ & $1.03(0.40-2.64)$ & $0.64(0.23-1.72)$ \\
2nd versus 1st tertile & & \\
3rd versus 1st tertile & &
\end{tabular}


TABLE 4 Adjusted odds ratios (aORs) ${ }^{\#}$ for the association between residential proximity to green space $(\leqslant 300 \mathrm{~m}$ versus $>300 \mathrm{~m})$ and health outcomes at the age of 4 years, stratified by biogeographic region.

\begin{tabular}{|c|c|c|c|}
\hline & All cohorts & Euro-Siberian & Mediterranean \\
\hline \multicolumn{4}{|l|}{ GEE model } \\
\hline Wheezing & 1754 & 721 & 1033 \\
\hline$\leqslant 300$ versus $>300$ & $0.92(0.75-1.13)$ & $0.67(0.45-0.99)$ & $0.83(0.66-1.06)$ \\
\hline Bronchitis & 1747 & 715 & 1032 \\
\hline$\leqslant 300$ versus $>300$ & $1.04(0.84-1.26)$ & $1.07(0.66-1.74)$ & $0.77(0.61-0.98)$ \\
\hline \multicolumn{4}{|c|}{ Logistic regression model } \\
\hline Asthma & 1771 & 724 & 1047 \\
\hline$\leqslant 300$ versus $>300$ & $0.60(0.31-1.18)$ & $0.99(0.36-2.68)$ & $0.60(0.25-1.45)$ \\
\hline Allergic rhinitis & 1769 & 722 & 1047 \\
\hline$\leqslant 300$ versus $>300$ & $0.67(0.34-1.30)$ & $0.63(0.22-1.77)$ & $0.84(0.36-1.94)$ \\
\hline \multicolumn{4}{|c|}{$\begin{array}{l}\text { Data are presented as } \mathrm{n} \text { or aOR }(95 \% \mathrm{CI}) \text { unless otherwise stated. \#:The pooled aORs (all cohorts) are } \\
\text { corrected for sex, cohort, maternal education, maternal smoking during pregnancy, any breastfeeding, } \\
\text { season of birth, maternal allergy, pets at home at birth, passive smoking at home at } 4 \text { years, and area } \\
\text { socioeconomic status (SES; according to the urban vulnerability index). The models stratified by } \\
\text { biogeographic region are corrected for all of the above except cohort and the generalised estimating } \\
\text { equation (GEE) model is additionally adjusted for time. }\end{array}$} \\
\hline
\end{tabular}

greenness and asthma in the pooled analysis was found to be stronger and attained statistical significance (3rd tertile versus 1st tertile: aOR 3.76, 95\% CI 1.17-12.07). Moreover, the positive relationship between higher exposure to residential surrounding greenness and asthma was more pronounced for the Euro-Siberian study region (2nd tertile versus 1st tertile: aOR 3.49, 95\% CI 1.17-10.40; 3rd tertile versus 1st tertile: aOR 3.22, 95\% CI 1.05-9.90) in children who had not changed residential address. For children from the Mediterranean study region, who were classified as more physically active $\left(\geqslant 12 \mathrm{~h} \cdot \mathrm{week}^{-1}\right)$, medium (but not high) exposure to residential surrounding greenness significantly reduced the risk for wheezing and bronchitis at age 4 years (2nd tertile versus 1 st tertile and 3rd tertile versus 1st tertile: aOR $0.59,95 \%$ CI $0.37-0.94$ and aOR $0.64,95 \%$ CI $0.41-0.99$, respectively). There were only minor changes for the association between exposure to higher residential surrounding greenness and wheezing for children

TABLE 5 Adjusted odds ratios (aORs) ${ }^{\#}$ for the association between exposure to residential surrounding greyness $\left(300-\mathrm{m}\right.$ buffer, $\left.\mathrm{m}^{2}\right)$ and health outcomes at the age of 4 years, stratified by biogeographic region.

\begin{tabular}{|c|c|c|c|}
\hline & All cohorts & Euro-Siberian & Mediterranean \\
\hline \multicolumn{4}{|l|}{ GEE model } \\
\hline Wheezing & 1777 & 729 & 1048 \\
\hline 2 nd versus 1 st tertile & $1.00(0.81-1.23)$ & $1.26(0.93-1.73)$ & $1.19(0.91-1.55)$ \\
\hline 3 rd versus 1 st tertile & $1.03(0.83-1.28)$ & $1.12(0.82-1.54)$ & $1.29(0.98-1.70)$ \\
\hline Bronchitis & 1770 & 723 & 1047 \\
\hline 2 nd versus 1 st tertile & $0.96(0.75-1.22)$ & $1.26(0.86-1.86)$ & $1.24(0.92-1.67)$ \\
\hline 3rd versus 1st tertile & $0.96(0.75-1.24)$ & $0.99(0.67-1.46)$ & $1.58(1.19-2.08)$ \\
\hline \multicolumn{4}{|l|}{ Logistic regression model } \\
\hline Asthma & 1771 & 724 & 1047 \\
\hline 2 nd versus 1 st tertile & $0.89(0.48-1.63)$ & $0.55(0.25-1.22)$ & $0.67(0.21-2.12)$ \\
\hline 3 rd versus 1 st tertile & $0.83(0.42-1.66)$ & $0.46(0.20-1.06)$ & $1.28(0.47-3.46)$ \\
\hline Allergic rhinitis & $n=1769$ & $n=722$ & $n=1047$ \\
\hline 2nd versus 1 st tertile & $0.79(0.40-1.58)$ & $0.87(0.35-2.13)$ & $1.35(0.50-3.68)$ \\
\hline $3 r d$ versus 1 st tertile & $1.00(0.50-2.01)$ & $0.43(0.16-1.18)$ & $1.26(0.45-3.50)$ \\
\hline \multicolumn{4}{|c|}{ 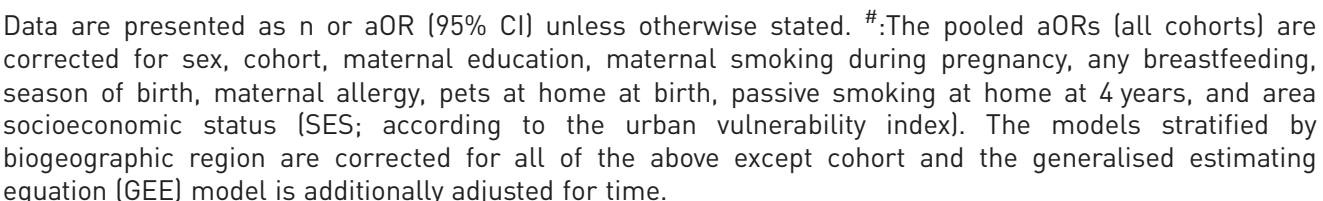 } \\
\hline
\end{tabular}


within the Euro-Siberian study area, taking account of physical activity status (less active children, 3rd tertile versus 1st tertile: aOR 0.51, 95\% CI 0.31-0.84; more active children, 3rd tertile versus 1 st tertile: aOR $0.65,95 \%$ CI $0.42-1.00)$.

\section{Discussion}

This is one of the first epidemiological studies to prospectively examine the impact of urban green and grey spaces on children's respiratory and allergic health using data from birth cohorts that span different bio-geographic regions. Higher residential surrounding greenness and proximity to green spaces were negatively associated with wheezing up to the age of 4 years in the Euro-Siberian region. In contrast, exposure to moderate, but not higher, residential surrounding greenness was associated with an increased risk for asthma among children growing up in this region. As we did not observe a clear dose-response pattern for this association, this finding needs to be interpreted with caution. We observed similar patterns of association for residential surrounding greenness in the Mediterranean region; however, none of the associations attained statistical significance. In this latter region, the risk of bronchitis was inversely associated with residential proximity to green spaces and positively associated with residential surrounding greyness.

To our knowledge, only a few prospective investigations (North America and Europe) have explored urban greenness in relation to respiratory outcomes. Tree canopy cover within a $250-\mathrm{m}$ buffer around the prenatal residential address was associated with an increased risk of asthma and specific allergic sensitisation among 7-year-old children living in New York City [32]. Among children living in the metropolitan area of Vancouver, exposure to higher surrounding greenness in a 100-m buffer around the home address in early life was negatively associated with incident asthma during preschool years ( $0-5$ years), but there was no association in later childhood (6-10 years) [9].

The impact of exposure to greenness on respiratory and allergic health might vary considerably. The protective effect appears to be especially pronounced in more rural areas or regions with a presumed higher biodiversity [33]. A prospective study in 2014 among children aged 10 years from the German LISAplus/GINAplus birth cohorts revealed heterogeneous effects of exposure to residential surrounding greenness (NDVI average) in relation to allergic outcomes across the two study centres (Munich-urban and Wesel-rural) included in the study's analysis [10]. FuerTes et al. observed that higher exposure to greenness was positively associated with allergic rhinitis, as well as eye and nose symptoms, among the more urban study area (Munich). However, among children living in the rural area (Wesel), exposure to greenness was inversely associated with these allergic outcomes. Potential protective effects of greenness in rural areas on asthma and allergies were partly confirmed by a recent meta-analysis, although overall, the effect estimates were highly heterogeneous across the seven study areas considered [13].

It has been speculated that the loss of natural environments and biodiversity, and the consequent decline in microbial diversity in living environments, might be partly responsible for the increase in the global prevalence of inflammatory diseases such as asthma and allergy in the rapidly urbanising world. Being exposed to a less diverse microbial environment in early life might lead to a weakened immune response or tolerance against harmful allergens ubiquitous in natural surroundings [1, 34, 35]. A recent study in Finland (2015) used land use patterns in buffers of size 2-5 km around the home to specifically test the "biodiversity hypothesis" (the loss of environmental biodiversity that emerges simultaneously with the increase in asthma and allergic outcomes) on atopic sensitisation [6]. Exposure to forest and agricultural land was inversely related to atopic sensitisation in children and adolescents aged up to 20 years. Within our study, we also observed a protective association between residential surrounding greenness and wheezing within the less urban Euro-Siberian region. However, we observed an association in the opposite direction for asthma within this same population. There might be several explanations for this discrepancy.

Although wheezing is recognised as a common symptom of asthma [36], it could also be due to non-allergic inflammatory responses of the airways caused by irritants from air pollution [37, 38]. Within our study, a higher amount of residential greenness in the Euro-Siberian region was associated with less air pollution $\left(\mathrm{NO}_{2}\right)$, as has been previously observed $[39,40]$. That could also explain why the effect of exposure to greenness in relation to wheezing was significant only for the Euro-Siberian study region (and not the Mediterranean area) and more pronounced when additionally adjusted for $\mathrm{NO}_{2}$ exposure. In two study centres (Sabadell and Gipuzkoa), asthma was assessed by a parent-reported diagnosis. In the other two centres (Asturias and Valencia), the parents only communicated the presence or absence of the disease. Unfortunately, we are unable to quantify the proportion of eventual misclassification caused by these different assessment methods.

The type (natural versus artificial) and quantity of urban green space may also be a decisive factor in its relation to respiratory health. Although participants living in the Euro-Siberian region were surrounded by 
denser vegetation than those in the Mediterranean region, Asturias and Gipuzkoa are also characterised by urban features. Compared with natural surroundings, artificial green urban areas and agricultural land use can also be potential sources of harmful allergen exposure [32, 41-43]. Species that are introduced into an area are likely to be non-native, and thus their consequences on allergic and respiratory health in the native population are unknown [44-46]. Fundamentally, it is likely that associations with respiratory health will depend on the allergenicity of the respective green exposure surrounding the participants [6, 44]. For instance, the forests of the Asturias region in the north of Spain are, to a large extent, characterised by Eucalyptus-monoculture (Eucalyptus globulus Labill) [47], which is originally native to Australia [48]. Similarly, Pinus radiata, a pine tree native to the Central Coast of California and Mexico, was introduced in the 19th century into Spain and is widely planted in the Basque Country of northern Spain [49]. These changes in the natural habitat might affect the diversity of the original natural environment, which may have implications for asthma and allergy [3]. Unfortunately, we did not have comprehensive information on the specific vegetation in the study areas and were unable to determine this using the exposure assessment methods currently available.

The relevance and interplay between both the outdoor and indoor environment could also be responsible for the heterogeneous results between and within our study centres. The "environmental microbiome" [34], the microbial profile dependent on the outdoor and indoor environment, and the human microbiome might differ considerably in levels, composition and diversity $[50,51]$ between and within urban and rural areas [52]. The traditional farm environment, which is associated with a higher and more diverse microbial exposure, is known to be strongly (protectively) associated with asthma and allergic diseases [34, 53]. To date, studies in urban areas looking at microbial exposure could only partly replicate these findings. It has been suggested that the relationship between the environmental microbiome and the development of allergic disorders might be more complicated in urban settings than in rural or native areas owing to a more heterogeneous exposure and abundance of other urban-related co-exposures [1]. In this context, the inconsistencies observed between our centres/regions might have also resulted from differences in cultural and behavioural patterns between the study areas. One recent publication underlined the importance of the relationship between the "inner" layer (the internal microbiota) and the "outer" layer (the outer microbiota) with respect to human health. The environmental microbial profile and its implications for health may be influenced by the individual use of green space, in terms of access as well as cultural and lifestyle deviations [54].

To our knowledge, this is the first study to investigate the impact of green space exposure on bronchitis as an indicator for respiratory infections in a paediatric population. We observed that residential proximity to green spaces was negatively associated with bronchitis at 4 years of age among children growing up in the Mediterranean study region. For these participants in this region, we also observed a similar inverse association between residential surrounding greenness and bronchitis after adjustment for $\mathrm{NO}_{2}$ exposure. Further, our study was the first to include a measure for residential surrounding greyness in order to better explore urban land use characteristics. A higher exposure to residential surrounding greyness was associated with a risk increase for bronchitis and wheezing (nearly statistically significant) for the participants in the Mediterranean region. In the only available study of the impact of urban land use on children's respiratory health, EBIsu et al. observed that a higher degree of urban land use, corresponding to a higher degree of urbanicity, was associated with greater severity of wheezing symptoms in infants [15], which is in line with our findings for wheezing and bronchitis up to the age of 4 years. These results suggest that the arrangement of green and grey spaces in the environment might considerably influence associated concentrations of allergens and air pollutant levels and, in turn, health outcomes [7, 55]. Unfortunately, using the data available, we cannot determine the underlying dimensions that describe residential surrounding greyness. Exposure specifically associated with urbanisation, such as noise, stress, heat islands or industry, may have unknown health consequences. Moreover, the degree of urbanisation may vary among regions and be influenced by cultural and behavioural factors $[54,56]$.

Our study has some limitations. Although our sample size was relatively large, the statistical power for the analyses of asthma and allergic rhinitis, especially in the stratified analyses, could have been limited, owing to the low prevalence of these conditions. This limitation also prevented us from evaluating other atopy-related conditions, such as allergic versus non-allergic asthma as well as allergic sensitisation. In addition, the health outcomes in the Sabadell and Gipuzkoa cohort were not assessed by a physician but recorded through parental self-reports. Further, we only had $\mathrm{NO}_{2}$ measurements for the first year of life. However, as the majority of the children did not change residential address until the age of 4 years, we can assume that their $\mathrm{NO}_{2}$ exposure was stable throughout the study period. We utilised remote sensing-derived NDVI to assess residential surrounding greenness. Application of this objective measure of greenness enabled us to take account of small-scale green spaces (e.g. home gardens, street trees and green verges) in a standardised way. However, the NDVI does not distinguish between different types of 
vegetation, which could be relevant for our investigated associations. Additionally, we had no data on severity, frequency and duration of episodes of allergic rhinitis. Lastly, there may be several reasons for the heterogeneous results, e.g. different regional, cultural, environmental and behavioural aspects. However, the assessment of these factors goes beyond the scope of our paper.

\section{Conclusion}

We evaluated the associations between three indicators of urban built environment and respiratory and allergic outcomes in children residing in two distinct bio-geographic areas. Several associations were found for respiratory health, but these differed in direction across the indices studied and by geographic region. These discrepancies in the results might be the consequence of the complex relationship between various factors, including the environmental microbiome in urban settings, which could not be assessed via the methods used in our study. Further research that considers region-specific characteristics at a finer scale, vegetation types and the distribution of native versus non-native vegetation is therefore warranted. Another appealing approach might be an integrative consideration of environmental characteristics by combining "green" and "grey" exposure using elaborated statistical methods.

\section{Acknowledgements}

The following are the funding bodies and awards that supported the work featured herein:

INMA Asturias: This study was funded by grants from the Instituto de Salud Carlos III (Red INMA G03/176 and CB06/02/0041), the Spanish Ministry of Health (FIS-FEDER PI042018, PI09/02311, and PI13/02429), the Obra Social Cajastur/Fundación Liberbank, and the University of Oviedo.

INMA Gipuzkoa: This study was funded by grants from the Instituto de Salud Carlos III (FIS-PI06/0867, FIS-PS09/ 00090, and FIS-PI13/02187), the Department of Health of the Basque Government (2005111093, 2009111069, and 2013111089), and the Provincial Government of Gipuzkoa (DFG06/004 and DFG08/001) (as annual agreements with the municipalities of the study area: Zumarraga, Urretxu, Legazpi, Azkoitia and Azpeitia, and Beasain).

INMA Sabadell: This study was funded by grants from the Instituto de Salud Carlos III (Red INMA G03/176) and the Generalitat de Catalunya CIRIT (1999SGR 00241). The 4-5 year follow-up was funded by grants from the Instituto de Salud Carlos III (Red INMA G03/176, CB06/02/0041, PI041436, and PI081151 incl. FEDER funds), the Generalitat de Catalunya CIRIT (1999SGR 00241), and the Fundació La marató de TV3 (090430).

INMA Valencia: This study was funded by Grants from the European Union (FP7-ENV-2011 cod 282957 and HEALTH.2010.2.4.5-1), the Instituto de Salud Carlos III (Red INMA G03/176, CB06/02/0041, FIS-FEDER 03/1615, 04/ 1509, 04/1112, 04/1931, 05/1079, 05/1052, 06/1213, 07/0314, 09/02647, 11/0178, 11/01007, 11/02591, 11/02038, 13/1944, 13/2032, 14/0891, and 14/1687) and the Conselleria de Sanitat, Generalitat Valenciana.

Christina Tischer is the recipient of a European Respiratory Society Fellowship (RESPIRE2-2015-7251). Elaine Fuertes is the recipient of a Marie Curie Individual Fellowship (MSCA-IF-2016). Payam Dadvand is funded by a Ramón y Cajal fellowship (RYC-2012-10995) awarded by the Spanish Ministry of Economy and Competitiveness.

\section{References}

1 Casas L, Tischer C, Täubel M. Pediatric asthma and the indoor microbial environment. Curr Environ Health Rep 2016; 3: 238-249.

2 Brodin P, Jojic V, Geo T, et al. Variation in the human immune system is largely driven by nonheritable influences. Cell 2015; 160: 37-47.

3 Hanski I, von Hertzen L, Fyhrquist N, et al. Environmental biodiversity, human microbiota, and allergy are interrelated. Proc Natl Acad Sci USA 2012; 109: 8334-8339.

4 Rook GA. Regulation of the immune system by biodiversity from the natural environment: an ecosystem service essential to health. Proc Natl Acad Sci USA 2013; 110: 18360-18367.

5 Mhuireach G, Johnson BR, Altrichter AE, et al. Urban greenness influences airborne bacterial community composition. Sci Total Environ 2016; 571: 680-687.

6 Ruokolainen L, von Hertzen L, Fyhrquist N, et al. Green areas around homes reduce atopic sensitization in children. Allergy 2015; 70: 195-202.

7 Dadvand P, Villanueva CM, Font-Ribera L, et al. Risks and benefits of green spaces for children: a cross-sectional study of associations with sedentary behavior, obesity, asthma, and allergy. Environ Health Perspect 2014; 122: $1329-1335$.

8 Dadvand P, de Nazelle A, Figueras F, et al. Green space, health inequality and pregnancy. Environ Int 2012; 40 $110-115$.

9 Sbihi H, Tamburic L, Koehoorn M. Greenness and incident childhood asthma: a 10-year follow-up in a population-based birth cohort. Am J Respir Crit Care Med 2015; 192: 1131-1133.

10 Fuertes E, Markevych I, von Berg A, et al. Greenness and allergies: evidence of differential associations in two areas in Germany. J Epidemiol Community Health 2014; 68: 787-790.

11 Lovasi GS, Quinn JW, Neckerman KM, et al. Children living in areas with more street trees have lower prevalence of asthma. J Epidemiol Community Health 2008; 62: 647-649.

12 Pilat MA, McFarland A, Snelgrove A, et al. The effect of tree cover and vegetation on incidence of childhood asthma in metropolitan statistical areas of Texas. HortTechnology 2012; 22: 631-637.

13 Fuertes E, Markevych I, Bowatte G, et al. Residential greenness is differentially associated with childhood allergic rhinitis and aeroallergen sensitization in seven birth cohorts. Allergy 2016; 71: 1461-1471.

14 Logan AC. Dysbiotic drift: mental health, environmental grey space, and microbiota. J Physiol Anthropol 2015; 34: 23.

15 Ebisu K, Holford TR, Belanger KD, et al. Urban land-use and respiratory symptoms in infants. Environ Res 2012; 111: 677-684. 
16 Guxens M, Ballester F, Espada M, et al. Cohort profile: the INMA-INfancia y Medio Ambiente (Environment and Childhood) Project. Int J Epidemiol 2012; 41: 930-940.

17 Mapa de series de vegetación de España. Madrid, Spanish Ministry of Agriculture, Food and Environment, 1987.

18 Dadvand P, Sunyer J, Basagaña X, et al. Surrounding greenness and pregnancy outcomes in four Spanish birth cohorts. Environ Health Perspect 2012; 120: 1481-1487.

19 Alcaraz-Segura D, Cabello J, Paruelo JM, et al. Use of descriptors of ecosystem functioning for monitoring a national park network: a remote sensing approach. Environ Manage 2009; 43: 38-48.

20 Dadvand P, Sunyer J, Alvarez-Pedrerol M, et al. Green spaces and spectacles use in schoolchildren in Barcelona. Environ Res 2017; 152: 256-262.

21 Weier J, Herring D. Measuring vegetation (NDVI \& EVI). http://earthobservatory.nasa.gov/Features/ MeasuringVegetation/ Date last updated: August 30 2000. Date last accessed: 24 March, 2017.

22 Donovan GH, Michael YL, Butry DT, et al. Urban trees and the risk of poor birth outcomes. Health Place 2011; 17: 390-393.

23 European Environment Agency. Urban atlas for Europe. www.eea.europa.eu/data-and-maps/ explore-interactive-maps/urban-atlas-for-europe Date last updated: April 06 2017. Date last accessed: May 042017.

24 European Commission Expert Group on the Urban Environment. Towards a local sustainability profile-European common indicators. Milan, Ambiente Italia, 2003. www.gdrc.org/uem/footprints/eci_final_report.pdf Date last accessed: May 042017.

25 European Environment Agency. CORINE land cover. www.eea.europa.eu/publications/COR0-landcover Date last updated: January 01 1995. Date last accessed: May 042017.

26 Hernández Aja A. Atlas of urban vulnerability in Spain 2001 and 2011: methodology, contents and credits. Madrid, Ministerio de Fomento, 2015. www.fomento.es/NR/rdonlyres/40668D5E-26B6-4720-867F286BD55E1C6B/135960/20160201METODOLOGIAATLASVULNERABILIDAD2001Y2011.pdf Date last accessed: May 042017.

27 Aguilera I, Pedersen M, Garcia-Esteban R, et al. Early-life exposure to outdoor air pollution and respiratory health, ear infections, and eczema in infants from the INMA study. Environ Health Perspect 2013; 121: 387-392.

28 Everitt BS, Hothorn T, A handbook of statistical analyses using R. Chapter 6: logistic regression and generalised linear models (blood screening, women's role in society, and colonic polyps). https://cran.r-project.org/web/ packages/HSAUR/vignettes/Ch_logistic_regression_glm.pdf Date last accessed: May 042017.

29 Halekoh U, Hojsgaard S, Yan J. The R package geepack for generalized estimating equations. J Stat Softw 2006; 15: $1-11$.

30 Yan J, Fine J. Estimating equations for association structures. Stat Med 2004; 23: 859-874.

31 R core team. The R project for statistical computing: getting started. www.r-project.org/ Date last accessed: May 04 2017.

32 Lovasi GS, O’Neil-Dunne JPM, Lu JWT, et al. Urban tree canopy and asthma, wheeze, rhinitis, and allergic sensitization to tree pollen in a New York City birth cohort. Environ Health Perspect 2013; 121: 494-500.

33 Ruokolainen L, Fyhrquist N, Haahtela T. The rich and the poor. Curr Opin Allergy Clin Immunol 2016; 16: 421-426.

34 von Mutius E. The microbial environment and its influence on asthma prevention in early life. J Allergy Clin Immunol 2016; 137: 680-689.

35 Rook GA, Lowry CA, Raison CL. Microbial “old friends", immunoregulation and stress resilience. Evol Med Public Health 2013; 46-64.

36 Asher MI, Keil U, Anderson HR, et al. International Study of Asthma and Allergies in Childhood (ISAAC): rationale and methods. Eur Respir J 1995; 8: 483-491.

37 Bono R, Romanazzi V, Bellisario V, et al. Air pollution, aeroallergens and admissions to pediatric emergency room for respiratory reasons in Turin, northwestern Italy. BMC Public Health 2016; 16: 722.

38 Götschi T, Heinrich J, Sunyer J, et al. Long-term effects of ambient air pollution on lung function: a review. Epidemiology 2008; 19: 690-701.

39 Nieuwenhuijsen MJ, Khreis H, Triguero-Mas M, et al. Fifty shades of green: pathway to healthy urban living. Epidemiology 2017; 28: 63-71.

40 Dadvand P, Nieuwenhuijsen MJ, Esnaola M, et al. Green spaces and cognitive development in primary schoolchildren. Proc Natl Acad Sci USA 2015; 112: 7937-7942.

41 DellaValle CT, Triche EW, Leaderer BP, et al. Effects of ambient pollen concentrations on frequency and severity of asthma symptoms among asthmatic children. Epidemiology 2012; 23: 55-63.

42 Bartra J, Belmonte J, Torres-Rodriguez JM, et al. Sensitization to Alternaria in patients with respiratory allergy. Front Biosci (Landmark Ed) 2009; 14: 3372-3379.

43 De Linares C, Belmonte J, Canela M, et al. Dispersal patterns of Alternaria conidia in Spain. Agric For Meteorol 2010; 150: 1491-1500.

44 Cariñanos P, Casares-Porcel M. Urban green zones and related pollen allergy: a review. Some guidelines for designing spaces with low allergy impact. Landsc Urban Plan 2011; 101: 205-214.

45 Belmonte J, Vilà M. Atmospheric invasion of non-native pollen in the mediterranean region. Am J Bot 2004; 91 : 1243-1250

46 Škarková P, Kadlubiec R, Fischer M, et al. Refining of asthma prevalence spatial distribution and visualization of outdoor environment factors using GIS and its application for identification of mutual associations. Cent Eur J Public Health 2015; 23: 258-266.

47 Cristina Zancada M, Almendros G, Jiménez Ballesta R. Humus quality after eucalypt reforestations in Asturias (Northern Spain). Sci Total Environ 2003; 313: 245-258.

48 Wrigley J, Fagg M. Eucalypts: a celebration. 2nd Edn. Crow’s Nest, Allen \& Unwin, 2013.

49 Cobos Suarez JM, Urrestarazu MMR. Forest health problems affecting Pinus radiata in Spain with special reference to the Basque region. New Zealand J For Sci 1989; 19: 228-230.

50 Pakarinen J, Hyvärinen A, Salkinoja-Salonen M, et al. Predominance of Gram-positive bacteria in house dust in the low-allergy risk Russian Karelia. Environ Microbiol 2008; 10: 3317-3325.

51 Täubel M, Rintala H, Pitkäranta M, et al. The occupant as a source of house dust bacteria. I Allergy Clin Immunol 2009; 124: 834-840. 
52 Weikl F, Tischer C, Probst AJ, et al. Fungal and bacterial communities in indoor dust follow different environmental determinants. PLoS One 2016; 11: e0154131.

53 Stein MM, Hrusch CL, Gozdz J, et al. Innate immunity and asthma risk in Amish and Hutterite farm children. $N$ Engl J Med 2016; 375: 411-421.

54 Ruokolainen L, Lehtimäki J, Karkman A, et al. Holistic view on health: two protective layers of biodiversity. Ann Zool Fennici 2017; 54: 39-49.

55 Shanahan DF, Fuller RA, Bush R, et al. The health benefits of urban nature: how much do we need? Bioscience 2015; 65: 476-485.

56 World Health Organization. Hidden cities: unmasking and overcoming health inequities in urban settings. Kobe, WHO Centre for Health Development, 2010. www.who.int/kobe_centre/publications/hiddencities_media/who_un_ habitat_hidden_cities_web.pdf Date last accessed: May 042017. 